\title{
The Effects of Positive Emotion Induced by the Autobiographical Emotional Memory Task Before Encoding on False Memory Formation in the Deese-Roediger-McDermott (DRM) Paradigm
}

\author{
Mervenur Ayyıldız \\ Department of Psychology \\ Marmara University \\ Istanbul, Turkey \\ ayyildizmervenur@gmail.com
}

\begin{abstract}
The relationship between emotions and false memories have been studied for many years. In particular, the importance of emotions in encoding has been revealed. In this study, the effects of positive emotion on false memory are investigated by inducing emotion before the learning task. 100 people between the ages of 18-50 without any psychological disorders were selected. Emotion induction was performed by Autobiographical Emotional Memory Task (AEMT) and happy emotion was induced by asking participants to write a happy memory. Deese-Roediger-McDermott (DRM) paradigm was used to examine the effect of positive emotion on false memory formation in neutral content. Participants watched a slide show including 3 neutral word lists of the DRM paradigm. Then, they were given a recognition test including the critical lures, control words, and the words in the slide show. It is observed that people in the neutral mood group made more mistakes in the recognition test than the happy mood group. This result may suggest that the effect of emotion on false memory might be more about the level of arousal, rather than the valence.
\end{abstract}

Key words: AEMT, emotion, happiness, DRM paradigm, false memory

\section{INTRODUCTION}

There is a communication between our memories, which can sometimes lead to false memory formation. The encoding of an event in the brain and its memory are two different things [1]. We usually interpret and relate what we see with our previous knowledge and memory. Therefore, by constructive and reconstructive processes, encoding and remembering happens [1]. These processes result in both true and false memories. Source monitoring framework proposes that during an event, some features bind together in our brain and we make attributions while we remember these events [1]. Sometimes emotion can promote these processes and increase people's tendency to have false memories. How an information is encoded is affected by different mood states induced before learning, and long-term memories and false memories are moderated by this process [2]. Several researchers examined the relationship between emotions and false memories by using the Deese-Roediger-McDermott (DRM) paradigm, in which every list is associated with a related word called "critical lure" [3]. This task is convenient for this type of research because critical lures are usually activated after seeing a presentation of a list of related words, and this can lead people during the recognition test to think they have seen the word [4].

The effects of emotions on memory can vary depending on whether they are given before or after encoding and depending on the content of the emotion. Emotion induction before encoding is the main focus in this research. The effects of different mood states differ between various studies. It is mostly because an event is encoded differently by positive and negative moods, and different memories are formed for the same event [5]. In addition, it is found that positive mood activates top-down processing, which results in more interpretation of the learned items, therefore more false memories [5]. On the other hand, they found that negative mood activates bottom-up processing, which in turn would result in more true memories because bottom-up processing does not require interpretation [5]. The difference between the effects of moods is also because while negative mood promotes item-specific memory, positive mood is believed to promote relational processing [7]. There are some studies investigating the different effects of valence on false memories. Valence is the content of the emotion, such as positive and negative [12]. We can consider happiness as a positive valence, and sadness as a negative valence. For example, one study suggested that sad mood supports item-specific processing, thus, the activation of critical lures in the DRM paradigm was reduced by the induction of negative mood prior to learning [2]. A very similar study also suggested that item-specific processing is supported by negative emotions [5]. They found that in the recall process, critical lures in the DRM paradigm are recalled less by the people in a negative mood, however, more by the people in a positive mood [5]. Similar results were also found for 
the recognition process [5]. Another study supports these and states that people in a happy or neutral mood showed more false memory effects than the people in negative moods [7]. Besides, according to the affect-as-information hypothesis, positive emotions encourage confidence in answers that are attainable or normative [6]. This usually results in relational processing, which causes the false memories [5]. Furthermore, in order to create item-specific processing in a positive mood, researchers tried another experiment, in which pictures and words were given jointly. They found that people in a positive and neutral mood were successfully performing item-specific processing [5]. Therefore, the false memory formation was reduced for these groups. However, no change was observed in participants with negative moods, and this finding proposes that people in a negative mood are already performing item-specific processing [5]. These results might be inferred that we focus on more specific things in the negative mood, while in the positive mood we associate the learned item with other information in our memory. Therefore, this idea suggests that happy people might be more prone to have false memories.

Although there are several studies about the effects of emotions on false memory, there is no study to my knowledge that examines the effects of the Autobiographical Emotional Memory Task (AEMT) on the Deese-Roediger-McDermott (DRM) paradigm. AEMT is a highly used method in order to induce the target emotion [8]. In this task, participants are asked to write about an emotional experience and it takes a very short time to perform it [8]. In this experiment, the effects of positive emotion induced by AEMT on the DRM paradigm including neutral words will be examined among people who are between the ages of 18 and 50, do not have any psychological disorder, and do not use any psychiatric medication. Similar to other results, in the recognition test of the DRM paradigm, I expect people in the positive emotion group to "miss" the seen neutral words more than the control group. Also, I expect people in the positive emotion group to say that they see words that they have not seen more than people in the control group, who are statistically less happy than the experimental group. Therefore, it is expected that happier people will give more false answers in the recognition test.

\section{METHODS}

\section{Participants}

Data were collected from individuals between the ages of 18 and 50. 127 people participated in the study. Participants were selected according to whether they had psychological disorders and whether they used psychiatric medications. Therefore, 27 of them were excluded because some of them were diagnosed with psychological disorders, some of them were using antidepressants or stimulants, and some of them did not write a happy memory that they were supposed to write. Therefore, 100 Turkish participants were included in the analysis. Half of them were in the experimental group and other half were in the control group. The mean age of the control group was $27,68(\mathrm{SD}=8,368)$, with a range from 19 to 50 . The mean age of the experimental group was $25,06(\mathrm{SD}=6,805)$, with a range from 18 to 45. Total participants' mean age was $26,37(\mathrm{SD}=7,701)$, with a range from 18 to 50 . In the experimental group, there were $6(12 \%)$ male and $44(88 \%)$ female participants. In the control group, there were 7 (14\%) male and $43(46 \%)$ female participants. In total, 87 $(87 \%)$ of the participants were female and $13(13 \%)$ of them were male.

\section{Materials}

\section{Demographic Questionnaire}

Participants were asked their age, gender, if they were diagnosed with a psychological disorder and its name, and if they use antidepressants or stimulants.

\section{Mood Check Before Manipulation}

Participants were asked to rate their current feeling, and a 9-point scale from very unhappy to very happy was used.

\section{Autobiographical Emotional Memory Task (AEMT)}

AEMT was used to manipulate emotional states, and participants in the experimental group were asked to write a happy memory in 5-7 sentences.

\section{Mood Manipulation Check}

After the mood induction, participants in the experimental group were asked to rate their current feelings for the second time, and a 9-point scale from very unhappy to very happy was used.

\section{Deese-Roediger-McDermott (DRM) Paradigm}

The Turkish version of the DRM paradigm [9] containing neutral items was used to see false memory formation. 3 lists of 13 words each were selected. 3 of these were critical lures, and 36 of them were the words that will be given in the slide show. A 36 second video was shown including 36 words, and each word was shown for 1 second. In the recognition task, participants were given randomized 21 words. 9 of them were from the video (e.g., wheel, dream, winter), 3 were the critical lures of those 3 lists (car, cold, and sleep), and 9 were control words taken from 3 different lists that were not shown in the video (e.g., cassette, skirt, computer) 
[9]. Participants were asked to answer each word from 1 (definitely did not see) to 7 (definitely saw). 1, 2, and 3 were accepted as "did not see", 4 was accepted as "do not remember", and 5, 6, and 7 were accepted as "saw". The words they saw in the video and said they saw were coded as "hits". The words they saw in the video but said they did not see were coded as "miss". The words they did not see in the video and said they did not see were coded as "correct rejection". The words they did not see in the video but said they saw were coded as "false alarm".

\section{Procedure}

Data was collected with an online form. Participants were assigned to the groups according to the month they were born. Those born in January-June were assigned to the control group, and those born in July-December were assigned to the experimental group. At first, participants were given a consent form. After that, they were asked to answer some demographic questions, then they rated their current emotional state from 1 to 9 . In order to create the target emotion, happiness, the participants in the experimental group were asked to write a happy memory of 5-7 sentences. Then, all participants rated their current emotional state for the second time to see if any change happened. Right after, 3 word lists of the Deese-Roediger-McDermott (DRM) paradigm were shown as a 36 second video, then they were immediately given 21 words as a remembering task.

\section{RESULTS}

The data was analyzed by using SPSS. Descriptive ANOVA was used to analyze the age and the gender of the control group, experimental group and the total participants. Descriptive ANOVA was also used in order to see the effect of AEMT on participants' emotions. Lastly, the Independent-Samples T Test was used to see the memory differences between control and experimental groups.

Descriptive ANOVA was carried out in order to see the emotional differences between the groups. It is found that in the first measurement of the current mood, the control group's mean score was 5,96 ( $\mathrm{M}=5,96$, $\mathrm{SD}=1,85$ ), and the experimental group's mean score was $5,7(\mathrm{M}=5,70, \mathrm{SD}=1,78)$. The experimental group's score increased in the second measurement $(\mathrm{M}=6,72$, $\mathrm{SD}=1,67)$. After this increase, the difference in emotion between the two groups was found to be statistically significant $[F(1,98)=4,66, p=0,033]$.

Independent Samples T-Test was used to see what memory differences there were between the two groups. First, there was no statistically significant difference in the number of not sure answers between the control group $(\mathrm{M}=0,82, \mathrm{SD}=1,30)$ and the experimental group $(\mathrm{M}=0,62, \mathrm{SD}=0,83) ; \mathrm{t}(98)=0,92, \mathrm{p}=0,069$. Second, there was a statistically significant difference in the number of missed words between the control group $(M=1,88$, $\mathrm{SD}=1,81)$ and the experimental group $(\mathrm{M}=1,54$, $\mathrm{SD}=1,16) ; \mathrm{t}(98)=1,12, \mathrm{p}=0,010$. Third, there was a statistically significant difference in the number of false alarms between the control group $(\mathrm{M}=3,2, \mathrm{SD}=2,2)$ and the experimental group $(\mathrm{M}=2,94, \mathrm{SD}=1,36) ; \mathrm{t}(98)=0,71$, $\mathrm{p}=0,050$.

\section{DISCUSSION}

In this experiment, it was hypothesized that people who are happier would produce more false memories for neutral words. However, the results demonstrated the opposite.

Firstly, the mood manipulation with AEMT was successful. The mean score of the experimental group's mood increased from 5,7 to 6,72. The mean score of the control group's mood was 5,96. Although they are close to the happy side, they might be considered to be more neutral than the other group.

Secondly, no significant difference was found between two groups in terms of "not sure" answers. However, the result was close to being statistically significant and when we look at the mean scores of both groups, it can be seen that the experimental group gave this answer less than the control group. This may indicate that happy people have more precise memories for neutral items in terms of whether they remember something or not.

Thirdly, the two groups were statistically different from each other in terms of the number of missed words. Missing the seen words means that they do not remember the words they saw. It is found that people in the happy condition gave more correct answers than the other group. This may indicate that happier people are better at recognizing neutral words they see.

Lastly, people in the happy condition were better at differentiating the critical lures and the control words from the seen words. Accordingly, it might be deduced that people with relatively neutral moods produced more false memories when remembering neutral words in this experiment, compared to happier people.

This experiment showed different results from the majority of the prior research. This might be because of several reasons. For example, some studies considered not only the valence but also the level of arousal. Arousal can be defined as how calming or exciting something is [12]. Anger can be given as an example of high arousal and sadness as an example of low arousal. Neutral mood is also considered as an example of 
low-arousal. One study considered happiness as a high-arousing effect and neutral mood as a low-arousing effect. They found that participants with a neutral mood demonstrated more false recognition of critical lures than happy participants [10], which is very similar to what is found in this experiment. Therefore, the finding in this experiment that happy people produce less false memory might also be related to arousal rather than valence. However, there are also some opposite findings. For example, one study found that high-arousal causes people to have more false memories regardless of the emotional state of the person [11]. According to this finding, they suggested that valence is not a necessary condition to see the effects of mood on false memory, and arousal could also be a factor [11]. Therefore, it is hard to make an interpretation about what is found in this experiment. There are different findings in the literature, and there might be several explanations for the results of this experiment. Thus, further research is needed.

This study had some limitations. First, the sample size was not enough and a bigger sample-size is needed for the further research. Second, more DRM lists could be used to be able to test more critical lures. Third, a similar experiment can be tried with words that contain emotion instead of neutral words. Besides, source memory was not tested but it might be a defining feature and more interpretation could be made. In addition, the control group was not given a filler task, and there might be some differences because of that. Future researchers might consider giving a filler task to the control group while the experimental group is given an emotion induction task and see if there are any differences this time. Furthermore, due to the ethical issues, negative mood induction could not be applied in this experiment. Lastly, future researchers might try to apply another emotion induction technique to see the differences between the results.

\section{CONCLUSION}

In conclusion, this is the first study investigating the effects of AEMT on DRM paradigm as far as I know. Differently from the majority of the previous research, this experiment showed that people with neutral moods, as opposed to happy people, made more mistakes in the recognition test of the DRM paradigm and showed more false memories for neutral words. This study is important because it might bring another perspective to the literature about the effects of happiness on false memories.

\section{ACKNOWLEDGEMENTS}

I would like to thank the people who participated in this experiment, Marmara University, and the instructor Dr. Özge Yllmaz for their contributions.

\section{REFERENCES}

[1] M. K. Johnson, C. L. Raye, K. J. Mitchell, E. Ankudowich, "The Cognitive Neuroscience of True and False Memories," in True and False Recovered Memories, Nebraska Symposium on Motivation, Vol. 58, R. F. Belli, Ed. New York, NY: Springer, 2012, pp. 15-52.

[2] J. Storbeck and G. L. Clore, "Affect influences false memories at encoding: Evidence from recognition data.," Emotion, vol. 11, no. 4, pp. 981-989, 2011.

[3] H. L. Roediger, and K. B. McDermott, "Creating false memories: Remembering words not presented in lists," Journal of experimental psychology: Learning, Memory, and Cognition, vol. 21, no. 4, pp. 803-814, 1995.

[4] J. D. Payne, L. Nadel, J. J. Allen, K. G. Thomas, and W. J. Jacobs, "The effects of experimentally induced stress on false recognition," Memory, vol. 10, no. 1, pp. 1-6, 2002.

[5] J. Storbeck, "Negative affect promotes encoding of and memory for details at the expense of the gist: Affect, encoding, and false memories," Cognition \& Emotion, vol. 27, no. 5, pp. 800-819, 2013.

[6] S. H. Bookbinder and C. J. Brainerd, "Emotion and false memory: The context-content paradox," Psychological Bulletin, vol. 142, no. 12, pp. 1315-1351, 2016.

[7] J. Storbeck and G. L. Clore, "With Sadness Comes Accuracy; With Happiness, False Memory: Mood and the False Memory Effect," Psychological Science, vol. 16, no. 10, pp. 785-791, 2005.

[8] C. Mills and S. D'Mello, "On the validity of the Autobiographical Emotional Memory Task for Emotion Induction," PLoS ONE, vol. 9, no. 4, pp. 1-10, 2014.

[9] M. Akdoğan, Ü. Akırmak, and İ. Gürsoy, "Türkçe Kelimelerin Sahte Anı üretme Oranlarının Deese-Roediger-McDermott (DRM) Paradigmasında incelenmesi," Türk Psikoloji Yazllarl, vol. 23, no. 46, pp. 31-51, 2020.

[10] I. Van Damme, "Mood and the DRM paradigm: An investigation of the effects of valence and arousal on false memory," Quarterly Journal of Experimental Psychology, vol. 66, no. 6, pp. 1060-1081, 2013.

[11] Y. Corson and N. Verrier, "Emotions and False Memories," Psychological Science, vol. 18, no. 3, pp. 208-211, 2007.

[12] E. A. Kensinger, "Remembering emotional experiences: The contribution of valence and arousal," Reviews in the Neurosciences, vol. 15, no. 4, pp. 241-252, 2004 